\title{
A Wide-Angle X-ray Diffraction Study of the Developing Embryonic Chicken Cornea
}

C. Boote, V. Siegler, K. M. Meek and A. J. Quantock

Biophysics Group, Dept. of Optometry and Vision Sciences, Cardiff University, Redwood Building, King Edward VII Avenue, Cardiff CF10 3NB, Wales UK.

Fibre Diffraction Review 11, 123-129, 2003

\section{ABSTRACT}

In terrestrial vertebrates the cornea is the main refractive component of the eye. Its remarkable mechanical toughness and almost 100\% light-transparency are largely a consequence of the unique collagenous architecture of the corneal stroma. We have used WAXS methods to investigate stromal remodelling in the embryonic chicken cornea in the latter stages of development. Collagen organisation at day 13-15 of embryogenesis is dominated by a four-fold orthogonal arrangement of fibrils. Thereafter this preferential alignment recedes, seemingly because further collagen is deposited in a more isotropic manner, masking the initial orthogonal template. In contrast, the mean lateral spacing of fibril-forming collagen molecules remains unaltered over this developmental period. Our observations have important implications for the biomechanical strength and shape of the cornea.

\section{Introduction}

The cornea is the tough, transparent window at the front of the eye. It serves as a physical barrier to mechanical trauma and infection, and provides around two-thirds of the eye's total focussing power. Instrumental to the cornea's function is the unique fibrous structure of the stroma, which constitutes the vast majority of the corneal thickness and is bounded on either side by thin epithelial and endothelial cell layers [1]. The bulk of the stroma comprises collagen fibrils that lie in layers, or "lamellae", in the plane of the cornea [2]. Fibrils within a single lamella lie parallel to each other, but are rotated with respect to fibrils in adjacent lamellae. The fibrils themselves are composed of rod-like Types I and V collagen molecules that run approximately parallel to the fibril axis and are spaced at regular $\sim 1.5 \mathrm{~nm}$ intervals. Collagen fibrils are strongest axially, and directions of preferred fibril orientation therefore closely associate with directions of increased tissue strength [3]. Tissue strength is paramount to the cornea's refractive function because of the need for the corneal surface to maintain its normal curvature under intraocular pressure.

Wide-angle equatorial diffraction from corneal stroma derives from interference between X-rays scattered from individual fibril-forming collagen molecules [4]. Because the molecules lie near-axially within fibrils, we can use the azimuthal (i.e. circumferential) intensity distribution in a corneal WAXS pattern to determine the relative number of fibrils oriented in any given direction (see Figure 1). Consider an X-ray beam of wavelength $\lambda$ incident on an isolated collagen fibril. We may represent the amplitude and phase of scattered X-rays by $\mathrm{F}(\mathbf{K})$, where $\mathbf{K}$ is related to the scattering angle $2 \theta$, by,

$$
|\mathbf{K}|=(4 \pi / \lambda) \sin \theta
$$

The resultant scattered intensity for an array of $N$ identical fibrils is given by:

$$
\mathrm{I}(\mathbf{K})=\sum_{j=1}^{N} \mathrm{~F}_{j}(\mathbf{K}) \mathrm{F}^{*}{ }_{j}(\mathbf{K})+\sum \sum_{j \neq k} \mathrm{~F}_{j}(\mathbf{K}) \mathrm{F}^{*}{ }_{k}(\mathbf{K}) \exp \left[\mathrm{i}\left(\mathbf{r}_{\mathrm{j}}-\mathbf{r}_{k}\right) \cdot \mathbf{K}\right]
$$

where $\mathbf{r}_{\mathrm{j}}$ defines the position of the $\mathrm{jth}$ fibril and $\mathrm{F}^{*}$ denotes a complex conjugate. The second term in Equation (2) represents interference between fibrils. However corneal collagen fibrils are arranged such that, $\left|\mathbf{r}_{\mathrm{j}}-\mathbf{r}_{\mathrm{k}}\right| \approx 60 \mathrm{~nm}[4]$, so that resulting diffraction fringes would be expected to appear for values of $|\mathbf{K}|$ of the order of $(2 \pi / 60) \mathrm{nm}^{-1}$. For our typical WAXS set-up, encompassing a $\sim 20 \mathrm{~cm}$ camera length, these fringes would (from Equation (1)) have a spacing at the detector of $\sim 5 \mu \mathrm{m}$. Such fringes do not make a significant contribution to the WAXS pattern when using a typical $200-500 \mu \mathrm{m}$ diameter X-ray beam. Thus wide-angle diffraction from an array of corneal collagen fibrils may be simply represented by the sum of intensities from individual fibrils, and 


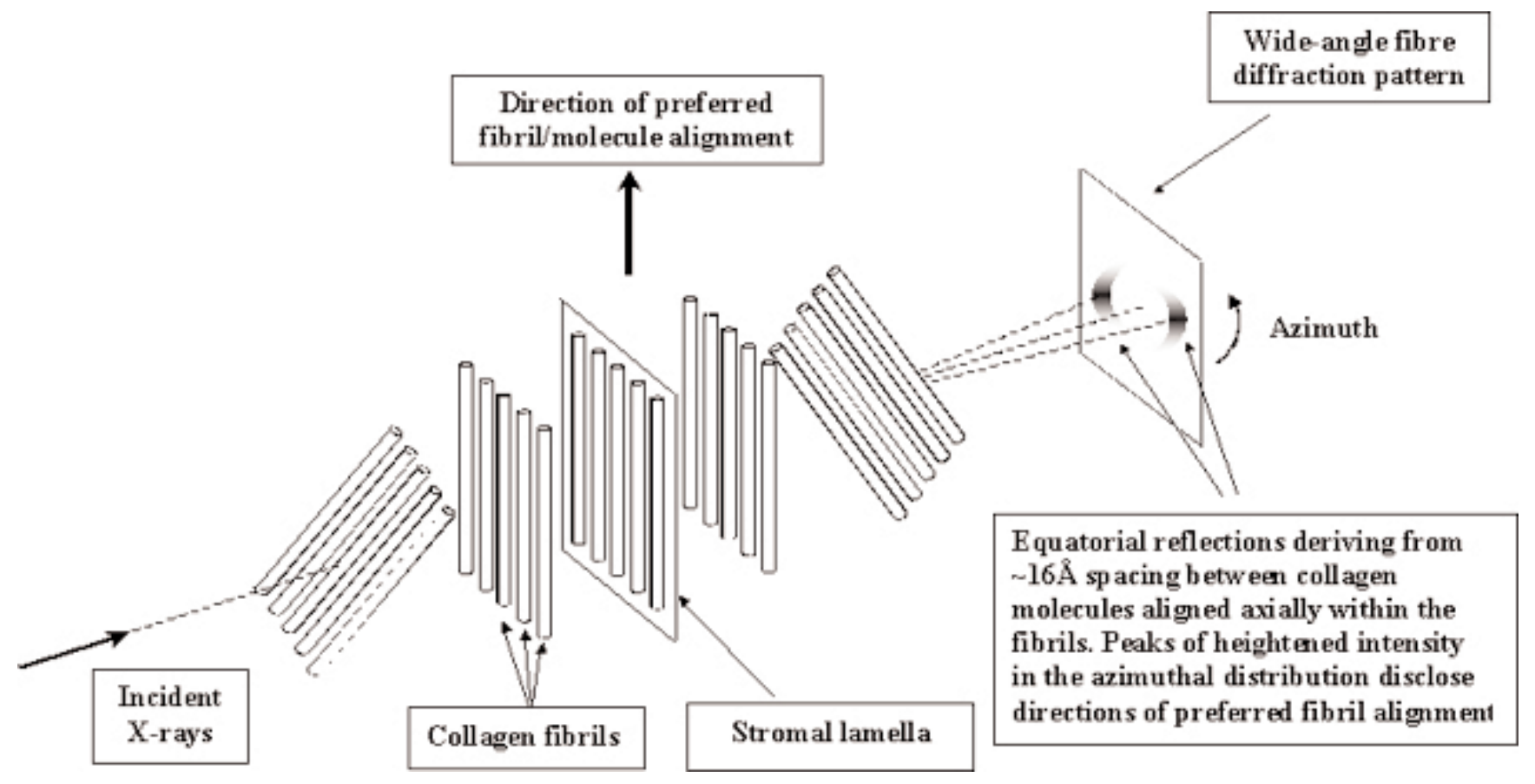

Figure 1: Wide-angle X-ray diffraction from corneal stroma.

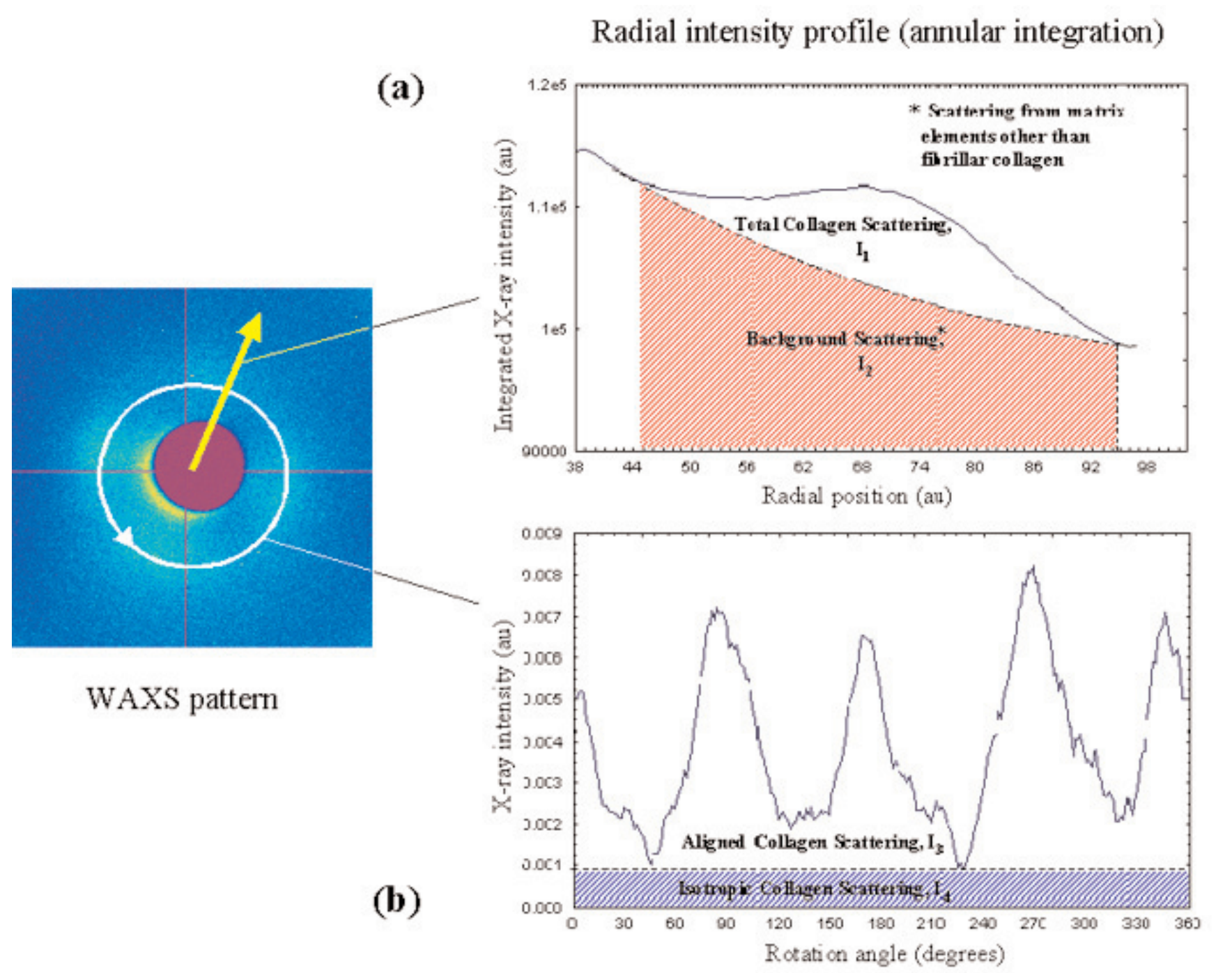

Annular intensity profile

Figure 2: Analysis of WAXS patterns from corneal stroma. (a) A radial intensity distribution allows the total fibrillar collagen to be expressed as the ratio of fibrillar collagen scattering to the total scattering from the stromal matrix i.e. $\mathrm{I}_{1} /\left(\mathrm{I}_{1}+\mathrm{I}_{2}\right)$. (b) An azimuthal intensity distribution allows the proportion of preferentially aligned collagen to be expressed as the ratio of scattering from aligned fibrils to scattering from all fibrillar collagen i.e. $\mathrm{I}_{3} /\left(\mathrm{I}_{3}+\mathrm{I}_{4}\right)$. 


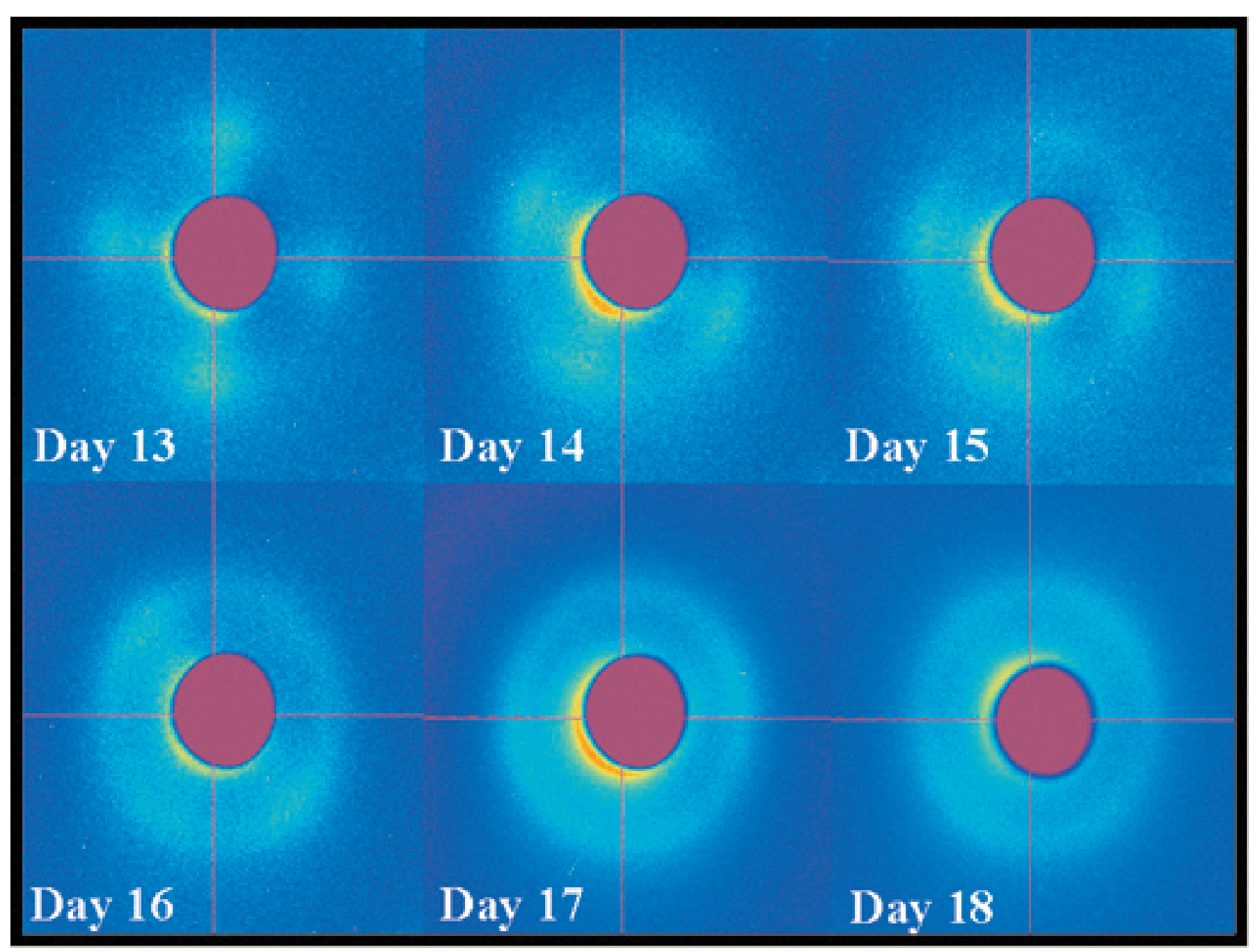

Figure 3: WAXS patterns from the centre of embryonic chicken corneas at developmental days 13-18. Reproduced from Quantock et al. (2002) [24], with permission of the copyright holder, the Association for Research in Vision and Ophthalmology.

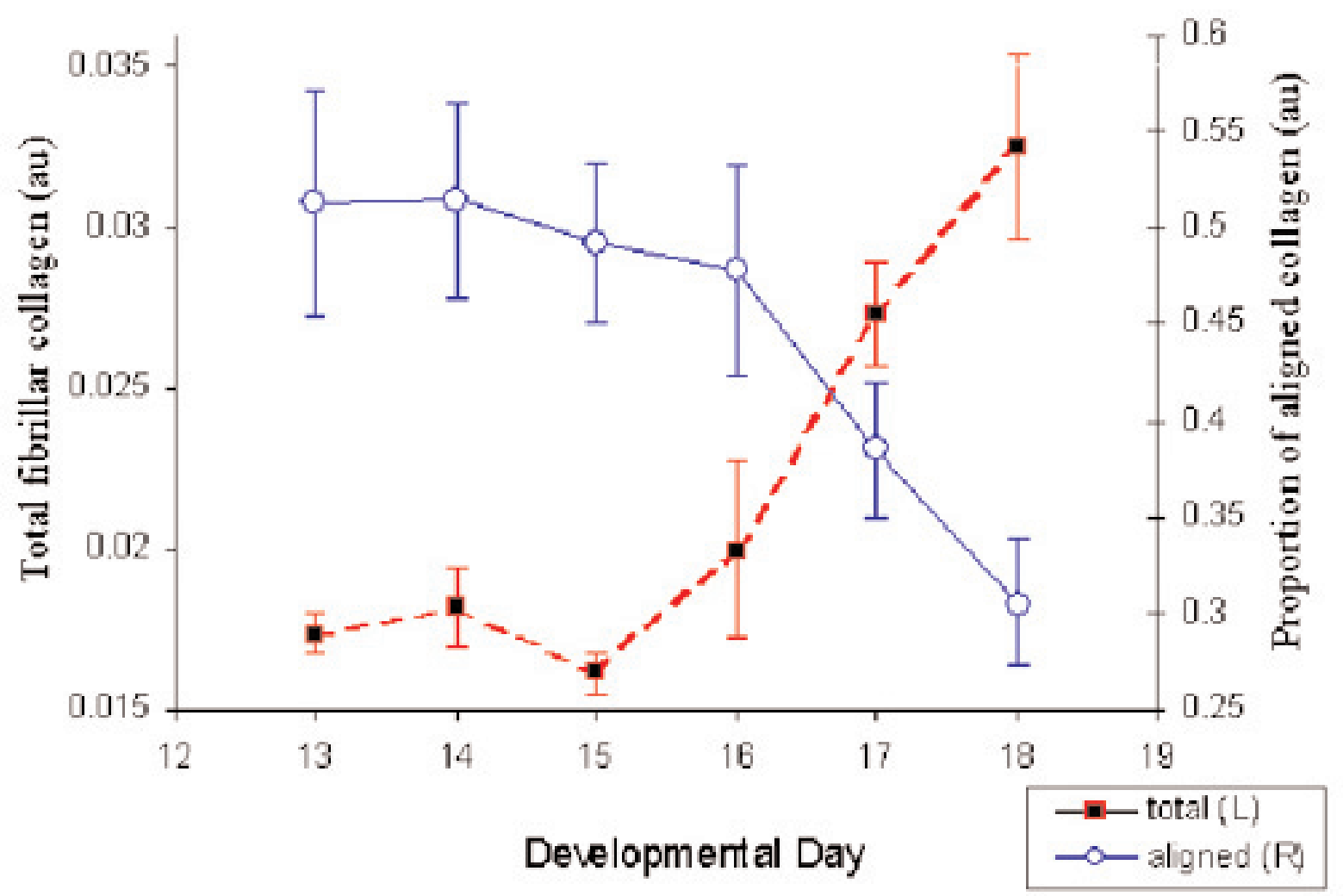

Figure 4: Variation in the total fibrillar collagen and proportion of aligned fibrillar collagen in embryonic chicken cornea at developmental days 13-18. 
interference between fibrils may be ignored, i.e.,

$$
\mathrm{I}(\mathbf{K}) \approx \sum_{\mathrm{j}=1}^{\mathrm{N}} \mathrm{F}_{\mathrm{j}}(\mathbf{K}) \mathrm{F}_{\mathrm{j}}^{*}(\mathbf{K})
$$

By extension, the $\mathrm{X}$-ray intensity is (to a first approximation) proportional to the number of aligned fibrils, and we define the azimuthal fibril distribution $\mathrm{B}(\phi)$ as,

$$
\mathrm{B}(\phi) \approx \mathrm{I}\left(\phi^{\prime}\right)
$$

where $\phi$ and $\phi$ ' are the azimuthal angles at the sample and detector respectively, and are related by

$\phi^{\prime}=\phi+90^{\circ}$. That is to say the scattering is equatorial, as shown in Figure 1. This approach to the analysis of WAXS data has been used previously to determine fibril orientations in other collagenous connective tissues $[5,6]$.

In the chicken, formation of the corneal stroma occurs as a sequence of distinct events initiated early in the 21-day incubation period, and beginning with the formation of the corneal epithelium [7]. The epithelial cells synthesise collagen fibrils that are secreted into the space between the corneal epithelial basement membrane and that of the lens directly posterior to this, so that by day 3 a loose $2-3 \mu \mathrm{m}$ thick network of collagen fibrils is formed known as the primary corneal stroma. On day 5 of embryogenesis the primary stroma is invaded by presumptive fibroblasts that continue to deposit collagen as the cornea matures into what is usually termed the secondary stroma.

Development of the secondary stroma from day 9 of embryogenesis until hatching at day 21 is of particular interest, because during this time period major remodelling of the stroma takes place that governs corneal function. Structural changes to both collagenous [8-11] and non-collagenous [12,13] stromal matrix components occur that are instrumental to the formation of a fully functional mature cornea. Most notable amongst these is a twostage fibril compaction in the stromal extracellular matrix, observed previously using SAXS techniques, that occurs during the latter stages of secondary corneal development $[8,10]$. Given that theories of corneal transparency require a degree of fibrillar order for light transmission [14], a link between fibril re-organisation and the acquisition of corneal transparency appears likely. However, the volume of literature describing the latter stages (i.e. after $\sim 14$ days) of secondary corneal development remains limited. In order to appreciate any reorganisation that might occur in the lateral spacing of fibril-forming collagen molecules and in the orientation of the corneal fibrils themselves during this important period of embryogenesis, we initiated a WAXS study.

\section{Experimental}

We collected fertilised hen eggs from a commercial hatchery and dissected out the corneas after 13, 14, 15, 16, 17 and 18 days of incubation. Excised corneas were immediately snap-frozen via immersion in liquid nitrogen-cooled isopentane, wrapped in Clingfilm to limit evaporation, and stored at $-80^{\circ} \mathrm{C}$ prior to the diffraction experiments.

Station 14.1 at the Daresbury SRS was used to collect WAXS patterns from a total of 47 corneas (n $=7$ or 8 at each developmental day). Each cornea was allowed to thaw within an airtight Perspex specimen chamber with Mylar entrance and exit windows. An X-ray beam of wavelength $0.1244 \mathrm{~nm}$ was passed through the centre of each cornea parallel to the optical axis. The beam dimensions at the sample measured $0.3 \mathrm{~mm} \times 0.3 \mathrm{~mm}$ and the exposure time was 2 minutes.

The experimental set-up was calibrated using the $0.304 \mathrm{~nm}$ X-ray reflection from powdered calcite. This achieved, we were able to determine the mean separation of fibril-forming collagen molecules in a central 300 micron zone of each cornea from the radial co-ordinate of the intermolecular X-ray reflection. In addition, the total intensity of this reflection provides a measure of the relative amount of fibrillar collagen present in the volume of tissue through which the X-ray passes. In order to generate a numerical index of this quantity, we generated radial distribution plots of scattered X-ray intensity, in which each point represented an integration through $360^{\circ}$ at that particular radius (see Figure 2(a)). The analysis protocol loosely followed that documented previously by Daxer and Fratzl [15]. A power law background function (representing scattering from stromal matrix elements other than fibrillar collagen) was fitted to and subtracted from the radial plots (Figure 2(a)), and the relative proportion of fibrillar collagen presented as the ratio of integrated intensity above background to the total integrated intensity (including background). This is equivalent to $I_{1} /\left(I_{1}+I_{2}\right)$ in Figure 2(a). 
As previously mentioned, the azimuthal distribution of X-ray intensity in the intermolecular reflection discloses the spread of fibril orientations present in the portion of tissue traversed by the X-ray beam. We produced azimuthal intensity plots for all 47 corneas studied, integrating radially over the intermolecular reflection. A numerical index of the relative amount of fibril alignment was computed as the ratio of $\mathrm{X}$ ray scatter from preferentially oriented fibrils to total scatter from all fibrillar collagen. This is denoted by $\mathrm{I}_{3} /\left(\mathrm{I}_{3}+\mathrm{I}_{4}\right)$ in Figure 2(b).

\section{Results}

Figure 3 shows a representative selection of WAXS patterns from corneas excised at developmental days 13 through 18. At days 13-15 the intermolecular reflection clearly displayed predominant four-fold symmetry, indicating the presence of a largely orthogonal fibrillar array. However, WAXS patterns from days 16-18 disclose a progressive recession in this arrangement, in favour of a more isotropic fibril array. A numerical index of the amount of preferentially aligned/orthogonal collagen was computed for all 47 corneas, and the mean values at each developmental time point are plotted in Figure 4. To more fully appreciate the structural remodelling at play here, we computed an index of the total fibrillar collagen present for each cornea and determined the mean value for each time point (Figure 4). The results disclose a significant increase in the deposition of fibrillar collagen, initiated at day

\begin{tabular}{|c|c|}
\hline $\begin{array}{c}\text { Developmental } \\
\text { Day }\end{array}$ & $\begin{array}{c}\text { Mean Intermolecular Bragg } \\
\text { Spacing, nm (S.D.) }\end{array}$ \\
\hline 13 & $1.42(0.03)$ \\
\hline 14 & $1.43(0.03)$ \\
\hline 15 & $1.42(0.02)$ \\
\hline 16 & $1.45(0.02)$ \\
\hline 17 & $1.41(0.02)$ \\
\hline 18 & $1.45(0.01)$ \\
\hline
\end{tabular}

Table 1: Mean intermolecular Bragg spacings for secondary embryonic chicken cornea at developmental days 13-18.

16 , which accompanies the loss of orthogonality. Analysis of the radial position of the intermolecular reflection provides a measure of the mean lateral separation of fibril-forming collagen molecules. The exact manner in which corneal collagen molecules pack into fibrils is still unknown, although studies of tendon have pointed to a possible quasi-hexagonal type packing arrangement [16]. Hence, the figures we present in Table 1 represent the mean intermolecular Bragg spacing for each developmental day. Conversion to centre-to-centre spacing requires multiplication by a packing factor, in the case of quasi-hexagonal packing this has been calculated to be 1.11 [17]. Our data reveal that the mean collagen intermolecular Bragg spacing remains unchanged at around $1.43 \mathrm{~nm}$ throughout the latter stages of secondary corneal development.

\section{Discussion}

As shown in Table 1, the mean separation of fibrilforming Type I/V collagen molecules does not change appreciably between days 13 and 18 of embryogenesis. The average value of $1.43 \mathrm{~nm}$ that we obtained is in the normal range and comparable to the mature avian cornea [18]. The result may at first appear surprising in light of the tissue dehydration that takes place during the same period [10], and which has been linked with the aforementioned fibril compaction. However, in vitro studies of the dehydration of bovine cornea $[19,20]$ reveal that, at the tissue hydration levels encountered in our study, water is removed almost exclusively from the interfibrillar space. Any intermolecular compaction would require water to be removed from within the fibrils themselves, a situation that only seems to occur at much lower tissue hydration.

Figure 4 shows that proportionally less collagen fibrils are aligned orthogonally as the embryonic cornea develops between days 13 and 18 of incubation. We believe that, rather than pointing to any degradation in the orthogonal matrix, this observation simply reflects the increased synthesis of collagen initiated at day 16 . The data provide evidence that the proportions of aligned and total fibrillar collagen respectively decrease/increase in a concomitant fashion (Figure 4). Based on current evidence it seems that, at day 16 , additional collagen is deposited isotropically so as to obscure the overall orthogonality of existing fibrils.

Let us consider how our observations can be reconciled with the established picture of the developing embryonic chicken cornea, gained mainly from light and electron microscopy observations. It has long been established that the primary corneal stroma at day 5 comprises an exclusively orthogonal array of fibrillar collagen 
synthesised by the epithelium [21]. Invading fibroblasts deposit collagen in register with the existing lamellae, thus preserving the orthogonality into the early stages of secondary corneal development. Consequently, the primary stroma is often considered the scaffold or template for the secondary cornea $[21,22]$.

Concurrently, the epithelium continues to synthesise collagen and deposit it anterior to the invaded stroma. This population of collagen, however, is deposited in lamellae that are successively rotated by about $3-6^{\circ}$ clockwise, as viewed from the endothelial side and traversing the cornea from its endothelial to epithelial surfaces [21]. As embryogenesis progresses, invasion of the expanding stroma by fibroblasts continues, with the fibroblasts continuing to lay down collagen in register with existing fibrils. Of course, because of the continuing deposition of rotated lamellae by the epithelium, the new collagen laid down by the fibroblasts is also rotated.

As a consequence of this dual mechanism of collagen deposition, by day 14 the secondary stroma has been shown to comprise a posterior $50 \mu \mathrm{m}$ layer containing mainly orthogonally arranged fibrils, with the remaining $150 \mu \mathrm{m}$ of anterior stroma being made up of progressively rotated lamellae exhibiting a net total angular displacement of $\sim 210^{\circ}$ [21]. If we assume that rotation between successive lamellae is fairly constant, a total net rotation of $180^{\circ}$ (or any multiple thereof) would represent a fully isotropic fibril distribution. Our contention is that the net rotation of $210^{\circ}$ estimated by Trelstad and Coulombre [21] at day 14 is close enough to $180^{\circ}$ to give rise to a fully homogeneous $\mathrm{X}$-ray reflection. Of course, since X-ray measurements represent an average through the full-thickness tissue, we expect the almost exclusively orthogonal posterior fibrils to produce a four-fold reflection, which would superimpose on the homogeneous reflection from the anterior stroma. This appears to be what we are observing at days 13-15 of development.

At day 16 there is clearly a swing towards a more isotropic fibril arrangement overall. While our data point to increased collagen deposition, rather than reorganisation or degradation of the posterior collagen template (Figure 4), it cannot, of course, tell us at which depth in the stroma this deposition might be occurring. However, in light of our data, it is interesting that immunofluorescence studies have revealed a dual layer of stable collagen in the anterior stroma that develops gradually between days 14 and 19, and which appears to form a physical link between the scleral ossicles (bony plates circumscribing the avian eye) [23]. It is possible that the collagen which we argue is deposited after day 16 , and acts to obscure the orthogonality in the posterior cornea, is associated with these two stable collagen bands. Furthermore, if, as has been suggested, the presence of the bands were required to stabilise the shape of the cornea [23], a totally isotropic arrangement of fibrils would clearly be an advantage biomechanically.

\section{Acknowledgements}

We would like to thank Mike MacDonald, Rob Kehoe and Mike Bailey for help at the SRS, Daresbury. This work was funded by a grant from the MRC (Programme Grant: 60001033) and the TFC Frost Charitable Trust.

\section{References}

[1] Forrester, J.V., Dick, A.D., McMenamin, P. \& Lee, W.R. (1996) The structure of the eye. In: The Eye.(Saunders, London).

[2] Maurice, D.M. (1957) The structure and transparency of the corneal stroma. J. Physiol. 136, 263-286.

[3] Hukins, D.W.L. (1996) Collagen fibril orientations in tissues and their relationship to mechanical properties. Fibre Diffraction Review 5, 14-16.

[4] Meek, K.M. \& Quantock, A.J. (2001) The Use of X-ray Scattering Techniques to Determine Corneal Ultrastructure. Prog. Ret. Eye Res. 20, 95-137.

[5] Boote, C., Sturrock, E.J., Attenburrow, G.E. \& Meek, K.M. (2002) Psuedo-affine behaviour of collagen fibres during the uniaxial deformation of leather. J. Mat. Sci. 37, 3651-3656. [6] Aspden, R.M. \& Hukins, D.W.L. (1979) Determination of the Direction of Preferred Orientation and the Orientation Distribution Function of Collagen Fibrils in Connective Tissues from High-Angle X-ray Diffraction Patterns. J. Appl. Cryst. 12, 306-311.

[7] Linsenmayer, T.F., Fitch, J.M., Gordon, M.K. et al. (1998) Development and roles of collagenous matrices in the embryonic avian cornea. Prog. Ret. Eye Res. 17, 231-265.

[8] Quantock, A.J., Kinoshita, S., Capel, M.C. \& Schanzlin, D.J. (1998) A synchrotron X-ray diffraction study of developing chick corneas. Biophys. J. 74, 995-998.

[9] Hirsch, M., Noske, W., Prenant, G. \& Renard, G. (1999) Fine structure of the developing avian corneal stroma as revealed by quick-freeze, deep-etch electron microscopy. Exp. Eye Res. 69, 267-277.

[10] Siegler, V. \& Quantock, A.J. (2002) Two stage compaction of the secondary avian cornea during development. Exp. Eye Res. 74, 427-431.

[11] Birk, D.E., Fitch, J.M. \& Linsenmayer, T.F. (1986) Organisation of collagen types $\mathrm{I}$ and $\mathrm{V}$ in the embryonic chicken cornea. Inv. Ophthalmol. Vis. Sci. 27, 1470-1477. 
[12] Cai, C.X., Gibney, E.P., Gordon, M.K., Marchant, J.K., Birk, D.E. \& Linsenmayer, T.F. (1996) Characterization and developmental regulation of avian corneal $\beta-1,4-$ galactosyltransferase mRNA. Exp. Eye. Res. 63, 193-200.

[13] Dunleavy, J.R., Beales, M.P., Berryhill, B.L., Cornuet, P.K. \& Hassell, J.R. (2000) Expression of the keratan sulfate proteoglycans lumican, keratocan and osteoglycin/mimecan during chick corneal development. Exp. Eye Res. 70, 349-362. [14] Farrell, R.A. (1994) Corneal transparency. In: Albert D.M. and Jacobiec S.A. (eds.) Principles and Practice of Ophthalmology. (Saunders, Philadelphia).

[15] Daxer, A. \& Fratzl, P. (1997) Collagen fibril orientation in the human corneal stroma and its implications in keratoconus. Inv. Ophthalmol. Vis. Sci. 38, 121-129.

[16] Fraser, R.D.B., MacRae, T.P., Miller, A. \& Suzuki, E. (1983) Molecular conformation and packing in collagen fibrils. J. Mol. Biol. 167, 497-521.

[17] Maroudas, A., Wachtel, E., Grushko, G., Katz, E.P. \& Weinberg, P. (1991) The effect of osmotic and mechanical pressures on water partitioning in articular cartilage. Biochim. Biophys. Acta. 1073, 285-294.

[18] Meek, K.M. \& Leonard, D.W. (1993) The ultrastructure of the corneal stroma: a comparative study. Biophys. J. 64, 273-
280.

[19] Meek, K.M., Fullwood, N.J., Cooke, P.H., et al. (1991) Sychrotron X-ray diffraction studies of the cornea with implications for stromal hydration. Biophys. J. 60, 467-474.

[20] Fratzl, P. \& Daxer, A. (1993) Structural transformation of collagen fibrils in corneal stroma during drying. Biophys. J. 64, 1210-1214.

[21] Trelstad, R.L. \& Coulombre, A.J. (1971) Morphogenesis of the collagenous stroma in the chick cornea. J. Cell Biol. 50, 840-858.

[22] Coulombre, J. \& Coulombre, A. (1975) Treatment of five-day-old embryos of domestic fowl with 6-Diazo-5-oxy-Lnorleucine (DON). Dev. Biol. 45, 291-303.

[23] Linsenmayer, T.F., Gibney, E. \& Fitch, J.M. (1986) Embryonic avian cornea contains layers of collagen with greater than average stability. J. Cell. Biol. 103, 1587-1593.

[24] Quantock, A.J., Boote, C., Siegler, V. \& Meek, K.M. (2003) Collagen Organisation in the Secondary Chick Cornea During Development. Inv. Ophthalmol. Vis. Sci. 44, 130-136. 